\title{
Les dynamiques du périurbain dijonnais entre 1990 et 1999
}

The dynamics of suburban Dijon between 1990 and 1999

Entwicklungsdynamik am Rand von Dijon zwischen 1990 und 1999

André Larceneux

\section{(2) OpenEdition}

Journals

Édition électronique

URL : http://journals.openedition.org/rge/1255

DOI : $10.4000 /$ rge. 1255

ISSN : 2108-6478

\section{Éditeur}

Association des géographes de l'Est

Édition imprimée

Date de publication : 1 janvier 2006

ISSN : 0035-3213

Référence électronique

André Larceneux, "Les dynamiques du périurbain dijonnais entre 1990 et 1999 », Revue Géographique de l'Est [En ligne], vol. 46 / 1-2 | 2006, mis en ligne le 17 décembre 2009, consulté le 08 septembre 2020. URL : http://journals.openedition.org/rge/1255 ; DOI : https://doi.org/10.4000/rge.1255

Ce document a été généré automatiquement le 8 septembre 2020

Tous droits réservés 


\section{Les dynamiques du périurbain dijonnais entre 1990 et 1999}

The dynamics of suburban Dijon between 1990 and 1999

Entwicklungsdynamik am Rand von Dijon zwischen 1990 und 1999

André Larceneux

\section{Introduction}

1 Comme la plupart des aires urbaines françaises, celle de Dijon a connu depuis les années soixante une très forte croissance. Ce mouvement a été accompagné d'un puissant processus de périurbanisation entraînant un développement sans précédent de l'étalement urbain. Associé à un accroissement des mobilités, cet étalement urbain est aujourd'hui assez généralement perçu comme incompatible avec des objectifs de développement durable. Impliquant majoritairement des déplacements automobiles, il apparait responsable aussi bien des encombrements routiers que des atteintes multiples à l'environnement : bruit, pollution et émission de gaz à effet de serre. Il se double d'un gaspillage de l'espace, mettant en cause l'agriculture au bénéfice de lotissements, de voies de communications et d'une artificialisation des sols responsable pour beaucoup d'une dégradation des qualités paysagères.

2 Les politiques urbaines ont souvent pour objectif affiché de limiter cette extension urbaine et de revenir à une ville plus compacte permettant une nouvelle dynamisation du transport collectif.

3 L'explication de l'étalement urbain, qui est la plupart du temps avancée, met en évidence le desserrement des centres et des banlieues par la mobilité résidentielle des ménages. Ceux-ci se déplaceraient vers les périphéries en raison des aménités rurales qu'elles offriraient, permettant une qualité de vie plus favorable que celle des centres urbains et surtout de leurs banlieues dégradées. L'habitat individuel périurbain aurait été une réponse efficace à la crise des banlieues, d'autant plus qu'il aurait été favorisé par les politiques publiques d'aide à l'accession à la propriété. Privilégiée par les classes 
moyennes et supérieures, la fuite des centres urbains et la recherche des aménités rurales auraient bénéficié de la baisse significative des coûts généralisés de transports. La construction de voies de communication et de radiales rapides a ainsi accru l'accessibilité des centres urbains et permis un accroissement de plus en plus grand des distances entre lieu de résidence et lieu d'emploi. Politique des transports et politique de l'habitat ont ainsi favorisé l'étalement urbain et le desserrement résidentiel.

Sans contester la pertinence de ce schéma, il convient sans doute de montrer qu'il n'explique pas la totalité du phénomène. En particulier, dans la période la plus récente, d'autres explications peuvent être trouvées qui mettent en avant des dynamiques de concentration des emplois. Cette concentration des emplois affaiblit les périphéries urbaines et leur croissance économique. Dans ces conditions, l'étalement urbain apparaît provoqué, non uniquement par un déplacement résidentiel, mais aussi par une mobilité forcée de recherche de travail. L'objectif de cette étude est de montrer l'imbrication de ces deux logiques sur l'aire urbaine dijonnaise. L'extension territoriale dijonnaise est manifeste mais ne concerne que l'aire urbaine.

\section{L'étalement urbain : définition, mesure et problème}

5 Dans plusieurs articles, Philippe Julien (Julien, 2000, 2001) a opportunément montré que la croissance urbaine pouvait être saisie à deux échelles différentes : d'une part, celle des pôles urbains, et d'autre part celle des aires urbaines qui nous concerne ici directement. Par ailleurs, il a mis en évidence que cet étalement pouvait être mesuré selon deux méthodes complémentaires. Il est possible d'analyser, dans un premier temps, les mouvements résidentiels sur un espace déterminé, autour d'un centre urbain (ou une aire urbaine) défini à une date donnée. Dans ce cas, le cadre géographique est fixé, par exemple en 1999, et on mesure la croissance de la population sur ce territoire entre plusieurs recensements.

Dans un deuxième temps il convient aussi d'étudier le phénomène dual, celui de l'extension proprement géographique des aires urbaines, agrégeant des communes de plus en plus éloignées du pôle.

7 Philippe Julien donne dans ses articles des résultats intéressants qui détaillent ces deux mouvements pour les plus grandes aires urbaines dont nous extrayons quelques données significatives.

Tableau 1 : Extension territoriale et croissance de la population de quelques aires urbaines

\begin{tabular}{|l|c|c|c|c|c|}
\hline Aire & $\begin{array}{c}\text { Population } \\
\text { 99 dans le } \\
\text { Territoire } \\
99(1)\end{array}$ & $\begin{array}{c}\text { Variation } \\
\text { dans le } \\
\text { Territoire } \\
\text { de 99 (2) }\end{array}$ & $\begin{array}{c}\text { Var. } \\
\text { totale } \\
\text { Ter.90 / } \\
\text { Ter.99 } \\
\text { (3) }\end{array}$ & $\begin{array}{c}\text { Var. 90/99 } \\
\text { de } \\
\text { l'extension } \\
\text { territoriale } \\
\text { (4) }\end{array}$ & $\begin{array}{c}\text { Population } \\
\text { 99 de } \\
\text { l'extension } \\
\text { territoriale } \\
\text { (5) }\end{array}$ \\
\hline Paris & 11175000 & 315084 & 882892 & 45362 & 613170 \\
\hline Lyon & 1648216 & 97083 & 140860 & 6777 & 50554 \\
\hline Strasbourg & 612104 & 43140 & 93625 & 4854 & 55339 \\
\hline
\end{tabular}




\begin{tabular}{|l|c|c|c|c|c|}
\hline Montpellier & 459916 & 71169 & 82503 & 2858 & 14192 \\
\hline Metz & 429588 & 12540 & 172024 & 691 & 160175 \\
\hline Nancy & 410508 & 4156 & 18517 & 408 & 14769 \\
\hline Dijon & 326000 & 13727 & 28641 & 519 & 15433 \\
\hline Besançon & 222381 & 14229 & 22771 & 995 & 9537 \\
\hline
\end{tabular}

Source : Recensements de la population

8 La taille des aires urbaine est ainsi dépendante de celle, préalable, des territoires « annexés » (colonne 5) par extension de leur surface géographique. La variation de la population dans le territoire de 99 ne doit pas être confondue avec la variation totale de l'aire urbaine $(\operatorname{col} 3=\operatorname{col} 2+\operatorname{col} 5-\operatorname{col} 4)$.

La deuxième des méthodes évoquées, qui se concentre sur l'accroissement géographique des aires urbaines, apparaît la plus pertinente pour notre propos. Elle nous conduit à chercher les raisons qui peuvent expliquer que des communes ont pu être rattachées au pôle urbain dijonnais entre 1990 et 1999. En effet, entre ces deux recensements, alors que le pôle urbain ne variait pas dans ses dimensions géographiques, 55 communes nouvelles ont été agrégées à l'aire urbaine dijonnaise expliquant en grande partie sa croissance globale. La population de l'aire urbaine a augmenté de 28641 habitants entre 90 et 99 dont 13727 seulement résultent de la variation dans les limites territoriales de l'aire urbaine en 99 (13 208 dans le territoire de 1990 et 519 dans l'extension territoriale). Le solde, soit 14914, correspond à la population en 1990 des communes agrégées. Ces communes nouvelles de l'aire urbaine ont eu une croissance démographique un peu plus faible que celle de l'aire sur le territoire de 1990 (3,4 \% contre 4,5\%) et que celle de l'ancienne périphérie (la couronne de 1990). Souvent même, ce qui peut surprendre, le solde migratoire de ces communes nouvellement intégrées est négatif. Ce faible dynamisme d'ensemble de ces communes fait écho à des difficultés importantes dans le domaine de l'emploi. Il convient donc de se pencher plus attentivement sur la situation de ces communes situées aux marges de l'aire urbaine.

La nécessité d'une telle étude a été suggérée par une publication récente de l'INSEE Bourgogne (M. Hilal, 2005). Elle montre des évolutions différentielles de l'emploi sur les espaces dijonnais. M. Hilal retient les territoires de l'intercommunalité : communauté d'agglomération, SCOT et aire urbaine (cartes 1 et 2), territoires qui intègrent un découpage institutionnel supplémentaire à l'intérieur même de la couronne périurbaine, celui du SCOT (Schéma de cohérence territorial). 
Carte 1 : Extension de l'aire urbaine dijonnaise entre 1990 et 1999. Le pôle urbain est resté inchangé.

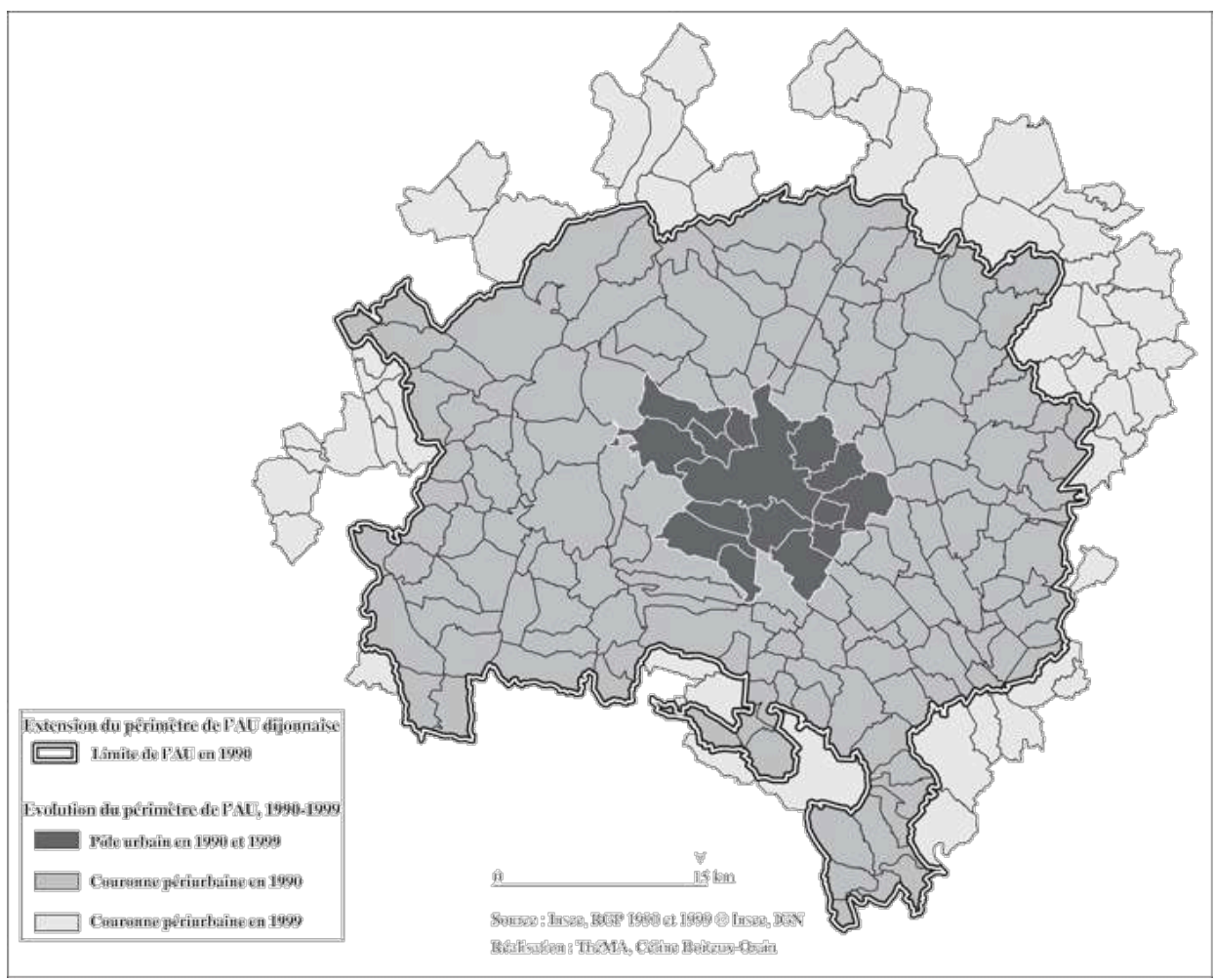


Carte 2 : Dynamique démographique des communes de l'aire urbaine dijonnaise 1990/1999

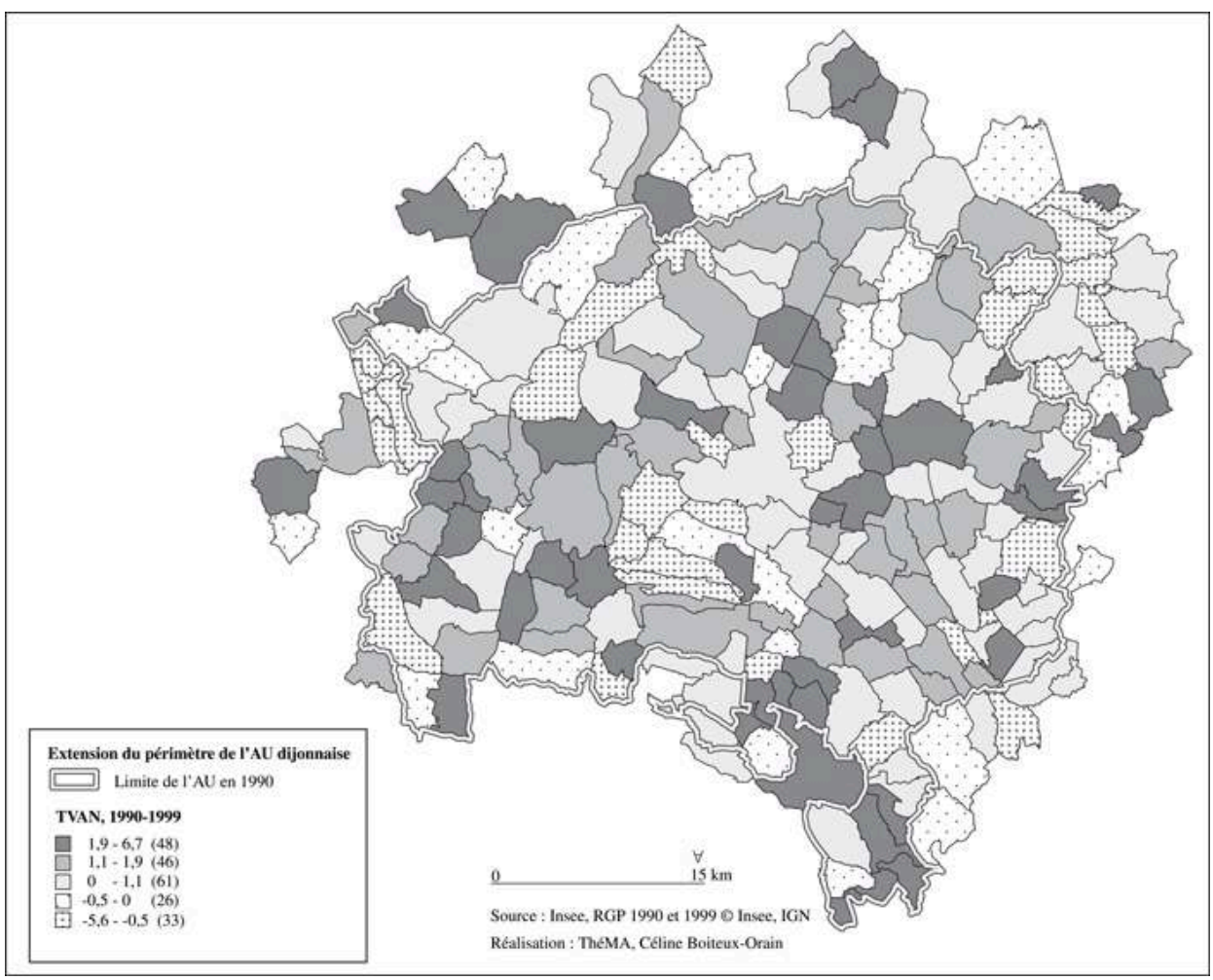

Source : Recensements de la population

Remarque : La communauté d'agglomération, le Grand Dijon, correspond à peu près au pôle urbain, il faut ajouter à ce dernier 6 petites communes qui appartiennent à l'aire urbaine. Le SCOT découpe la couronne périurbaine en retenant les communes proches du Grand Dijon.

11 La baisse de l'emploi est donc sensible sur les zones les plus périphériques (aire urbaine hors SCOT), alors même que leur intégration dans l'aire urbaine dijonnaise est récente. L'étude de M. Hilal montre de plus, que l'emploi occupé par des personnes résidant dans ce même territoire a très fortement reculé $(-20,7 \%$ soit près de 800 emplois en moins) alors que les emplois occupés par des personnes résidant en dehors de cette zone, notamment hors de l'aire urbaine dijonnaise, ont augmenté de plus de 450 . Les mobilités de travail semblent être liées pour partie dans ce cas à la baisse de l'emploi et à la nécessité de rechercher du travail dans le pôle urbain ou à sa proximité. Ces données indiquent aussi une très forte croissance des mobilités et une généralisation des déplacements domicile-travail.

Tableau 2 : Variations de l'emploi dans l'aire urbaine dijonnaise (Délimitation de 1999)

\begin{tabular}{|l|c|c|c|}
\hline Emploi & 1990 & 1999 & $99 / 90$ \\
\hline Grand Dijon & 115554 & 120974 & 4,6 \\
\hline SCOT hors GD & 12634 & 13454 & 6,5 \\
\hline Aire urbaine hors SCOT & 4665 & 4353 & $-6,7$ \\
\hline
\end{tabular}




\begin{tabular}{|l|l|l|l|}
\hline - dont actifs résidants & 3663 & 2903 & $-20,7$ \\
\hline
\end{tabular}

Source : M. Hilal, 2005

La situation particulière de ces communes nouvellement intégrées à l'espace dijonnais présente donc un réel intérêt et il convient de se focaliser sur leur propre dynamique : peut-on mettre en évidence pour ces communes, d'une part un mouvement continu et de plus en plus éloigné de desserrement résidentiel et d'autre part une dynamique de recherche d'emploi vers le pôle urbain ? L'accroissement général des mobilités semble alors s'accompagner d'un délitement relatif de l'emploi périphérique et d'une réorganisation des pôles secondaires. Des pôles nouveaux sont apparus, attirant une nouvelle population, alors que d'autres espaces en régression ont dû voir leurs populations résidantes se diriger vers des pôles plus centraux. Ce phénomène, sensible sur les couronnes périphériques internes de l'aire urbaine, se fait aussi sentir sur des espaces encore extérieurs : près de $10 \%$ des emplois de l'aire urbaine dijonnaise sont occupés par des actifs résidant à l'extérieur de cette aire. Parmi ceux-ci, plus de la moitié travaillent dans la commune même de Dijon. Les dynamiques de localisation de l'emploi sont donc incontournables dans l'explication de la périurbanisation et de l'extension des aires urbaines.

\section{L'extension de l'aire urbaine de Dijon entre 1990 et 1999}

13 Entre les deux derniers recensements, 55 communes nouvelles ont été rattachées à l'aire urbaine dijonnaise (carte 3). Elles se situent normalement sur les périphéries, à l'exception du Sud-Est où l'extension est limitée par le relief et l'absence de voies de communication. La petite commune isolée de Bouhey est en fait reliée par la vallée de l'Ouche. Parmi ces communes, on compte deux anciens pôles ruraux, Mirebeau sur Bèze et Brazey en Plaine qui dépassent les mille habitants (respectivement, 1573 et 2457 habitants). L'extension est la plus marquée à l'est dans la vallée de la Saône et au nord. L'étalement de l'aire urbaine bute sur d'autres pôles ruraux qui résistent encore à leur intégration. Même si pour ces derniers, en valeur absolue, de nombreux migrants alternants travaillent dans l'aire urbaine, ces communes se maintiennent comme pôles d'emplois relatifs. Le nombre des migrants alternants n'atteint pas le seuil des $40 \%$ des actifs résidants : ce sont Nuits-St-George, St Jean-de-Losne, Auxonne, Pontailler, Is-surTille et St Seine-l'Abbaye. Dans ces deux derniers cas, situés au nord de l'aire urbaine, les nouvelles communes intégrées à l'aire urbaine forment une sorte d'encerclement progressif de ces pôles et donnent une allure curieusement découpée au nouveau territoire. Les principaux pôles ruraux, intégrés ou non, se situent sur des axes de communication (nationaux ou départementaux) qui se dirigent en étoile vers Dijon. 
Carte 3 : Evolution de l'emploi des nouvelles communes de l'aire urbaine dijonnaise

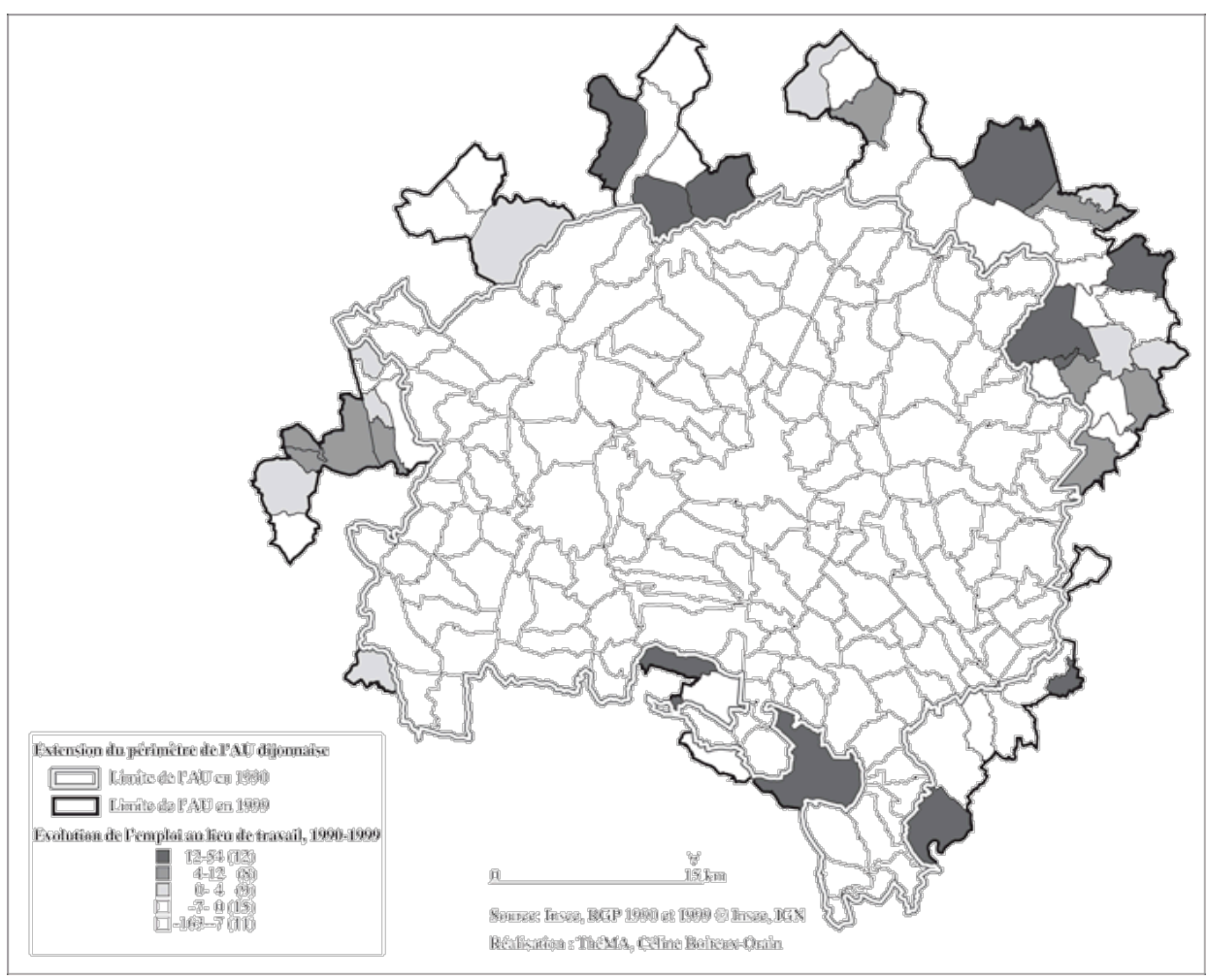

14 Ces nouvelles communes de l'aire urbaine ont connu des croissances très différenciées entre 1990 et 1999. Plusieurs, 24 sur 55, ont perdu de la population dont cinq plus de $15 \%$. Il n'apparaît pas de très nette tendance géographique, communes en croissance démographique et communes en décroissance se côtoyant assez fortement. Si les communes de la côte viticole sont toutes en progression, le sud-est avec Brazey en Plaine enregistre de nets reculs comme l'ouest, alors que dans le nord les dynamiques positives l'emportent. Aucune logique d'ensemble ne se dégage à l'est, en dépit de la croissance du pôle que constitue encore Mirebeau. Mais les autres communes proches ont des évolutions très divergentes. Ainsi Drambon et Saint Sauveur ont des croissances qui dépassent $20 \%$ alors que Saint Léger et Montmançon qui leur sont contiguës reculent sensiblement.

Les deux pôles ruraux ont des évolutions contradictoires. Le plus important, Brazey-enPlaine, perd $1,7 \%$ de sa population, soit $-0,19 \%$ par an avec un solde migratoire annuel négatif de $-0,78 \%$. A l'inverse, Mirebeau-sur-Béze croît de plus de $7 \%$ sur la période soit annuellement de 0,8\% avec un solde migratoire positif de $1,21 \%$. Curieusement, ces deux pôles ont des croissances naturelles elles aussi opposées, mais positive pour Brazey et négative pour Mirebeau. De la même manière, les évolutions de leur emploi sont contrastées : alors que Mirebeau connaît une croissance soutenue (+3\%), Brazey en Plaine enregistre un net recul (-15\%).

Tableau 3 : Evolution de la population et de l'emploi 1990-1999 des communes selon la croissance de la population ou de l'emploi

\begin{tabular}{|c|c|c|c|c|}
\hline & $\begin{array}{c}\text { Population } \\
1999\end{array}$ & $\begin{array}{c}90 / \\
99\end{array}$ & $\begin{array}{c}\text { Emploi au lieu de } \\
\text { travail 1999 }\end{array}$ & $\begin{array}{c}90 / \\
99\end{array}$ \\
\hline
\end{tabular}




\begin{tabular}{|l|c|c|c|c|}
\hline Mirebeau & 1573 & 1,09 & 651 & 1,03 \\
\hline Brazey & 2457 & 0,98 & 580 & 0,85 \\
\hline $\begin{array}{l}\text { Toutes comm. en } \\
\text { croissance* }\end{array}$ & 9330 & 1,09 & 1816 & 1,25 \\
\hline $\begin{array}{l}\text { Toutes comm. en } \\
\text { décroissance* }\end{array}$ & 6103 & 0,95 & 1635 & 0,77 \\
\hline Total & 15433 & 1,03 & 3451 & 0,97 \\
\hline
\end{tabular}

* Les communes ne sont pas les mêmes selon la population ou l'emploi.

Source : Recensements de la population

16 Ainsi, les communes qui font désormais partie de l'aire urbaine dijonnaise ont des configurations dynamiques très diverses tant par la croissance de leur population que par celle de leur emploi.

17 La croissance de la population est dans tous les cas observés liée à un solde migratoire positif, ce qui pourrait s'interpréter comme la conséquence d'un desserrement résidentiel, les nouveaux arrivants provenant de la ville-centre ou de sa banlieue. Toutefois, il peut s'agir aussi d'un rapprochement vers des pôles d'emploi dynamiques pour des populations résidant auparavant à l'extérieur de l'aire urbaine et attirées par un emploi plus central. Le mouvement mesuré n'est bien évidemment qu'un solde de déplacements complexes et ne peut être réduit à un mouvement simple du centre vers la périphérie. Il faut le replacer dans l'ensemble des flux migratoires résidentiels. 
Carte 4 : Typologie des communes périphériques

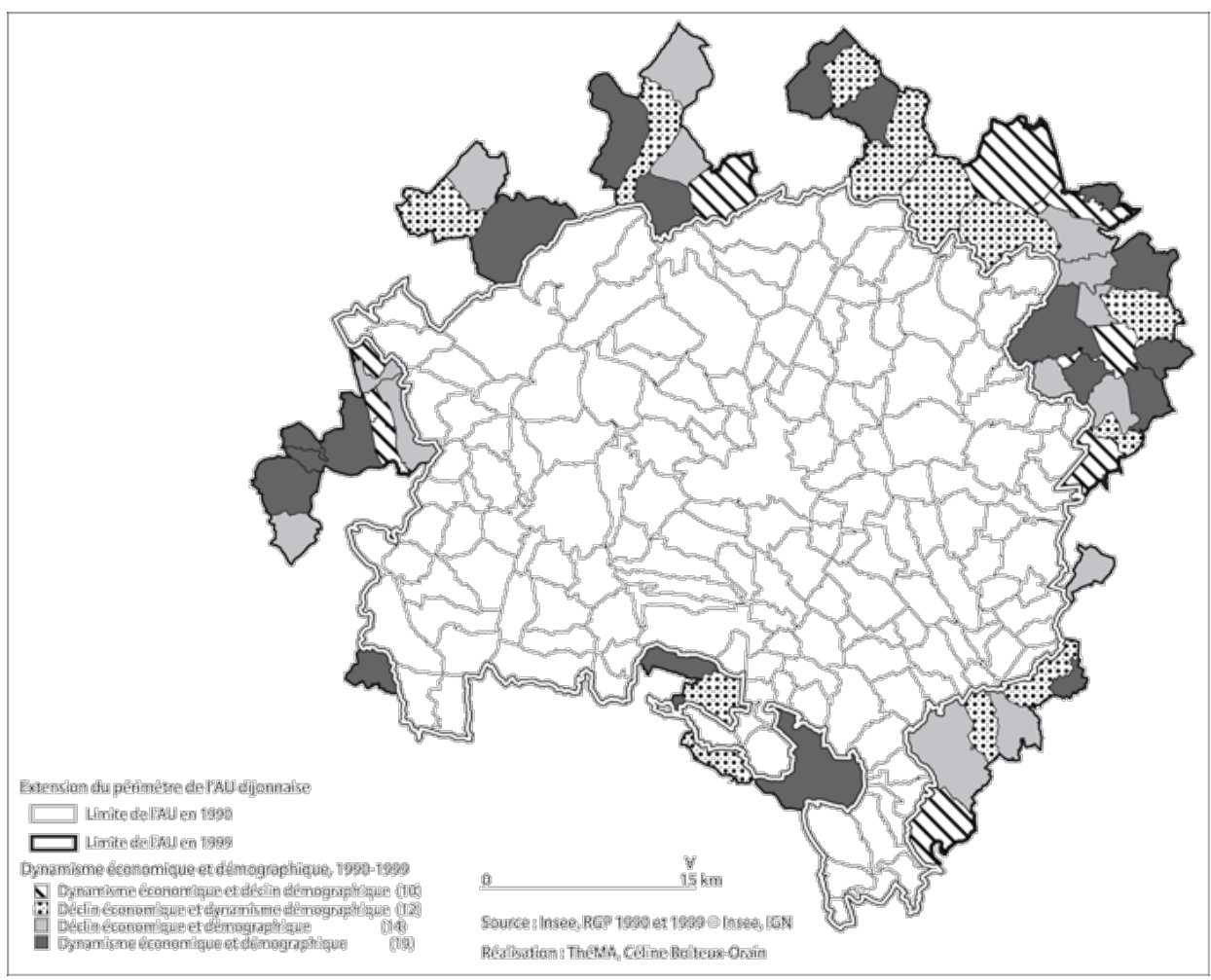

18 Les communes qui semblent le plus nettement et directement attirées par l'aire urbaine de Dijon ont une population en croissance et se développent pour beaucoup, semble-til, indépendamment du pôle rural près duquel elles se situent. Ces pôles, notamment Issur-Tille au nord et Nuits-St-Georges au sud, sont eux-mêmes progressivement et de plus en plus dépendants de Dijon et du reste de l'aire urbaine ; s'ils résistent encore comme pôles secondaires d'emploi, ils ne structurent plus les communes de leur environnement, passées dans l'orbite dijonnaise. On peut s'attendre, si le mouvement de périurbanisation devait encore s'étendre, à ce que ces pôles suivent l'évolution déjà ancienne de Genlis, Gevrey-Chambertin ou Sombernon ou récente de Brazey et Mirebeau et qu'ils s'intègrent à l'aire urbaine.

Tableau 4 : Dynamisme économique et démographique des communes intégrées à l'AU de Dijon entre 1990 et 1999

\begin{tabular}{|l|l|l|l|}
\hline & & \multicolumn{2}{|c|}{ Population } \\
\hline & & Croissance & Décroissance \\
\hline
\end{tabular}




\begin{tabular}{|c|c|c|c|}
\hline \multirow{2}{*}{ Emploi } & Croissance & $\begin{array}{l}\text { Bouhey, Champagne- } \\
\text { sur-Vingeanne, } \\
\text { Charmes, Echevannes, } \\
\text { Grosbois-en-Montagne, } \\
\text { Jancigny, Licey-sur- } \\
\text { Vingeanne, Marcellois, } \\
\text { Mirebeau-sur-Bèze, } \\
\text { Moloy, Morey-Saint- } \\
\text { Denis, Pont, Saint- } \\
\text { Mesmin, Saint-Nicolas- } \\
\text { les-Citeaux, Saint- } \\
\text { Sauveur, Vaux-Saulnes, } \\
\text { Vernot, Villey-sur-Tille et } \\
\text { Vougeot }\end{array}$ & $\begin{array}{l}\text { Bezouotte, Bourberain, } \\
\text { Champrenault, } \\
\text { Cheuge, Dampierre-et- } \\
\text { Flée, Drée, Esbarres, } \\
\text { Saint-Léger-Triey, } \\
\text { Verrey-sous-Drée et } \\
\text { Villecomte }\end{array}$ \\
\hline & Décroissance & $\begin{array}{l}\text { Bèze, Boncourt-le-Bois, } \\
\text { Champdôtre, } \\
\text { Courtivron, Crecey-sur- } \\
\text { Tille, Drambon, Gilly- } \\
\text { les-Citeaux, Lux, } \\
\text { Montot, Poncey-sur- } \\
\text { l'Ignon, Renève et Til- } \\
\text { Chatel }\end{array}$ & $\begin{array}{l}\text { Beaumont-sur- } \\
\text { Vingeanne, } \\
\text { Blagny-sur-Vingeanne, } \\
\text { Brazey-en-Plaine, } \\
\text { Bussy-la-Pesle, Civry- } \\
\text { en-Montagne, } \\
\text { Cuiserey, Magny- } \\
\text { Montarlot, } \\
\text { Montmancon, Oisilly, } \\
\text { Pellerey, Poiseul-les- } \\
\text { Saulx, Saint-Helier, } \\
\text { Tarsul et Trouhans }\end{array}$ \\
\hline
\end{tabular}

19 Ainsi, et cela peut sembler paradoxal, de nombreuses communes en recul démographique ont été intégrées à l'aire urbaine. Ce recul s'explique dans tous les cas par une évolution négative du solde migratoire. Ces communes n'attirent donc pas de populations nouvelles: leur intégration urbaine ne peut venir de nouveaux arrivants, recherchant des zones présentant des aménités rurales et quittant les zones plus urbanisées. Certes là aussi, le taux de migration étant un solde, cela n'interdit pas qu'un taux négatif soit obtenu avec des arrivées effectives de populations résidantes compensées par des départs massifs.

20 Ainsi, des communes se rattachent à l'aire urbaine dijonnaise alors même qu'elles sont en déclin démographique. Peu attractives du point de vue résidentiel, ces communes ont néanmoins des évolutions d'emplois différenciées. Comment et pourquoi, dès lors, peuvent-elles se rattacher à l'aire urbaine?

Certaines communes sont directement intégrées dans l'aire urbaine, en raison des changements qu'elles manifestent. Les actifs de celles qui perdent des emplois ont pu en trouver un nouveau dans une autre commune de l'aire urbaine, définie dans ses limites de 1990. Pour celles qui enregistrent en même temps une croissance de l'emploi et une décroissance de la population, l'explication est à chercher dans l'accroissement général des mobilités associé à un problème d'appariement sur le marché du travail, les emplois locaux ne correspondant pas aux qualifications de la population résidante. Pour celles qui voient population et emploi diminuer, le délitement général pousse à la migration. 
Par ailleurs, des communes peuvent aussi être placées dans l'aire attraction d'autres communes, en particulier des deux pôles secondaires, qui ont connu une intégration à l'aire urbaine de Dijon. Par effet pur de structure et de cascade et en raison des modes de construction statistique des aires urbaines, ces communes, même si elles n'ont connu aucun changement, ont pu se retrouver comptées désormais dans l'espace périurbain dijonnais.

\section{Migrations définitives d'actifs et d'inactifs}

Les dynamiques territoriales mettent en jeu une tension entre migrations définitives et alternantes. Quels sont les déplacements quotidiens qui sont encore acceptables et à partir de quel moment doit-on changer de résidence? Cette tension s'exerce sur les deux pôles que représentent le domicile et le travail. L'attrait pour d'éventuelles aménités rurales et la centralité de l'emploi sont les deux bornes de cette tension. S'éloigner de son travail ou s'en rapprocher peuvent expliquer tout autant les migrations définitives. Les périphéries sont traversées par ces deux formes de migrations résidentielles. Mais si les migrations d'actifs occupent une place importante, elles n'expliquent pas tous les mobilités: celles des inactifs ne doivent pas être occultées et ne se rapportent donc pas à la construction des aires urbaines.

Carte 5 : Mouvements migratoires et âge des migrants

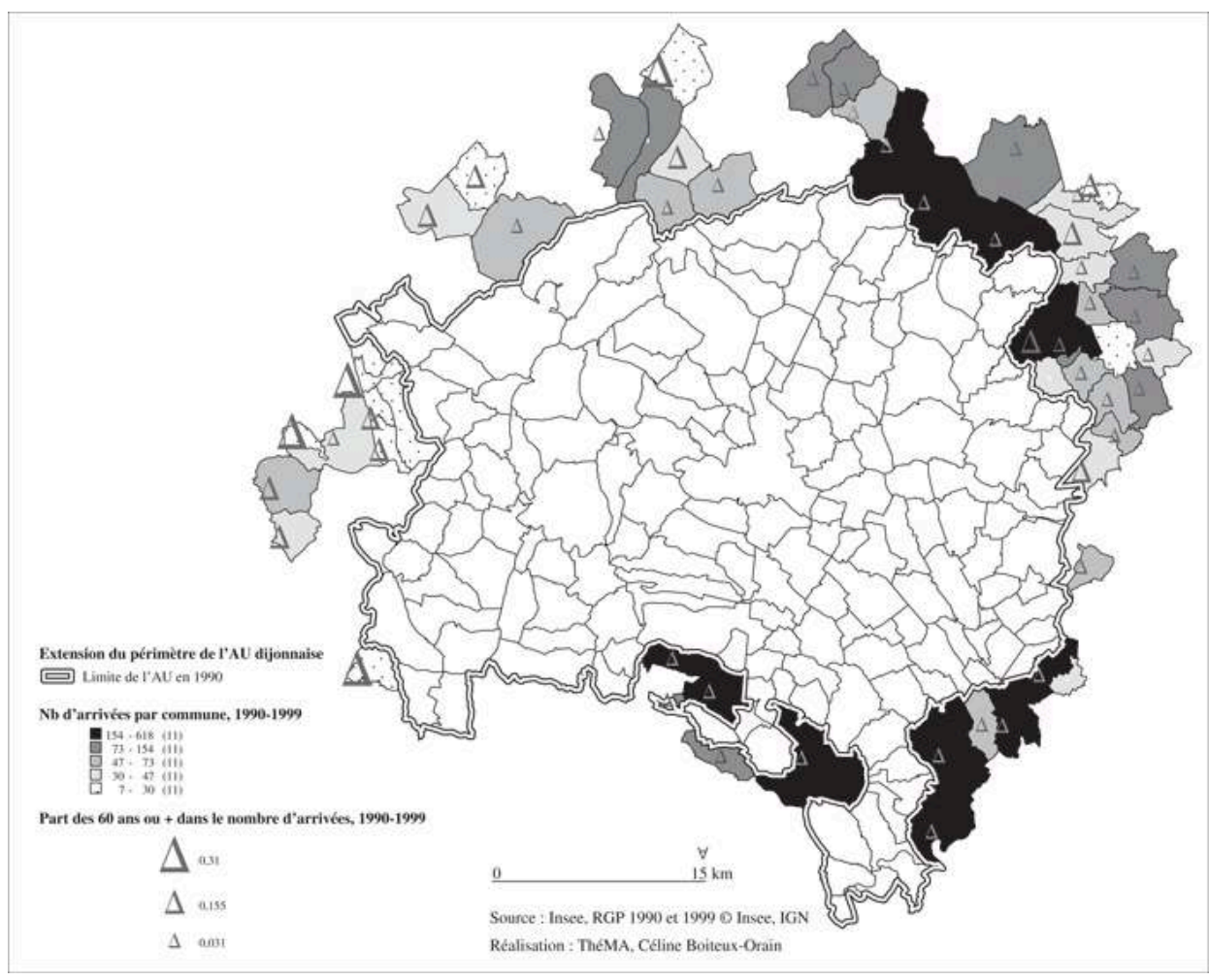

Sources : Recensements de la population

La croissance de la population due au solde migratoire positif n'est pas en soi un gage de l'intégration des communes dans l'aire urbaine. En effet, si cette migration est le fruit d'inactifs, notamment de retraités, elle ne modifie pas les flux alternants. Néanmoins, elle peut affecter les mobilités vers les pôles de services de l'agglomération 
et se traduire par une dépendance des territoires vis-à-vis du pôle urbain ou de la commune centre. Pour un nombre important des communes nouvelles de la périphérie dijonnaise, le pourcentage des personnes de plus de 60 ans dans les nouveaux arrivants dépasse $30 \%$. Il faudrait sans doute voir dans ces mouvements migratoires une des explications importantes du desserrement résidentiel. Mais celui-ci est assez nettement déconnecté des migrations alternantes bien qu'il puisse apparaître lié à des périodes du cycle de vie et à des retours dans des habitations de famille. Ces mouvements sont bien évidemment impossibles à saisir avec les seules données des recensements. Mais ils contribuent à disjoindre les mouvements résidentiels et les déplacements pour l'emploi.

\section{Conclusion}

L'étalement urbain se révèle être un phénomène beaucoup plus complexe qu'il ne paraissait à première vue. Si l'étalement géographique du pôle urbain dijonnais est contenu, aucune commune nouvelle ne s'y agrège entre 1990 et 1999, la croissance de l'aire urbaine s'est poursuivie, intégrant des communes de plus en plus éloignées. Les dynamiques résidentielles ont joué un rôle indéniable, quoiqu'il soit difficile de discriminer entre un véritable desserrement résidentiel et les choix de localisation des nouveaux arrivants. Mais c'est sans doute dans les difficultés économiques rencontrées par les communes périphériques, les pertes d'emplois et les recompositions territoriales qu'il faut aussi trouver les raisons de l'extension de l'aire urbaine dijonnaise. Les mouvements de l'emploi, leur concentration dans le pôle urbain mais aussi leur développement certes plus modeste dans certaines communes de la périphérie, sont responsables des reculs observés dans nombre des communes nouvelles de l'aire urbaine. Ces mouvements sont responsables d'une part importante de la croissance des mobilités et de l'extension des aires urbaines. Cet étalement urbain n'est alors que la manifestation, révélée par la définition conventionnelle des seuils de mobilité, de la concentration des emplois dans le pôle urbain ou même dans la ville centre. Ce phénomène semble du reste concerner en priorité les communes les plus éloignées.

Il serait vain de prétendre contraindre et limiter l'étalement urbain en ne se concentrant que sur l'habitat et les pratiques résidentielles. Les dynamiques de l'emploi central, favorisées le plus souvent par les politiques urbaines et les stratégies des acteurs locaux dominants, ont incontestablement une part importante de responsabilité. En d'autres termes, pour promouvoir des politiques de développement durable, contrôler les flux de mobilité et encourager la poursuite de la concentration des emplois dans les pôles urbains semblent des objectifs assez nettement contradictoires. Une meilleure connaissance des marchés du travail, de leur organisation et de leur spécialisation est, au contraire, nécessaire pour progresser dans la maîtrise des déplacements urbains, sources importantes de nuisances et de pollutions. 


\section{Sur quelques définitions : les pôles et les aires urbaines}

La délimitation du territoire en aires urbaines, initiée par l'Insee, a permis une meilleure compréhension de l'accroissement de l'influence des villes. Cette délimitation conventionnelle, désormais bien connue, distingue deux ensembles dont les logiques de construction sont différentes, la première porte sur l'agglomération du bâti pour les pôles urbains et la seconde, sur les mobilités pour définir les périphéries périurbaines.

Les pôles urbains sont définis parmi les unités urbaines à partir de deux critères :

1. ils recouvrent des agglomérations morphologiques du bâti, formées sur le critère habituel de proximité $(200 \mathrm{~m})$;

2. ils sont formés généralement d'une commune ville-centre et d'une banlieue ;

3. ils doivent atteindre une taille de 5000 emplois.

29 Les couronnes périurbaines moins denses sont construites à partir des déplacements domicile-travail au sein de cette aire urbaine (et non en direction du seul pôle urbain). Un seuil de mobilité est fixé : $40 \%$ des actifs ayant un emploi dans une commune doivent travailler dans une autre commune de l'aire urbaine.

Ces définitions qui permettent d'efficaces comparaisons sur l'ensemble du territoire national présentent des lacunes lorsqu'elles sont appliquées à un espace particulier. On peut signaler quelques limites impliquées par ces définitions.

1. Un pôle non urbain est ainsi défini comme rural: compte tenu du seuil fixé, une unité urbaine de près de 10000 habitants peut se trouver ainsi « ruralisée ».

2. Les mobilités sont définies en pourcentage et non en valeurs absolues. Ainsi, des communes très petites avec peu d'emplois peuvent être intégrées dans l'aire urbaine, alors qu'un pôle « rural » voisin, qui envoie un flux conséquent de personnes dans l'aire urbaine, en sera exclu.

3. Les communes formant une aire urbaine sont agrégées selon un principe itératif: ainsi, l'attraction réelle du pôle peut être surestimée. Plusieurs communes, notamment d'anciens pôles ruraux désormais intégrés, peuvent être des intermédiaires dans le processus retenu, et les communes classées dans l'aire urbaine n'avoir qu'un très lointain rapport avec le pôle urbain lui-même.

31 Enfin, la structuration unique en termes de relation domicile-travail évacue un peu rapidement l'influence des pôles de services dont, il est vrai, la connaissance est plus difficile. De même, les constructions purement statistiques des espaces entretiennent des relations complexes avec les structures institutionnelles en plein développement qui se mettent en place dans le cadre de l'intercommunalité.

Tout changement des seuils et des principes de construction retenus entraînerait, bien évidemment, une modification des territoires faisant partie des aires urbaines. Il faut se souvenir que les définitions retenues sont conventionnelles. 


\section{BIBLIOGRAPHIE}

Atlas de l'aire urbaine dijonnaise (2002). - Laboratoire ThéMA Dijon.

Hilal M. (2005). - « Localisation des emplois et déplacements domicile-travail dans le Grand Dijon et sa périphérie » Dimensions INSEE Bourgogne.

Julien P. (2000). - « Mesurer un univers en expansion » Economie et Statistique $\mathrm{n}^{\circ} 336$ INSEE.

Julien P. (2001). - « Les grandes villes françaises étendent leur influence » INSEE n 766.

Larceneux A. (2005). - « Franges de métropoles : l'extension périfrancilienne de Paris » Colloque ASRDLF septembre 2005.

Merlin P. (1998). - « Les banlieues des villes françaises » La documentation française.

Ministère de l'Aménagement du Territoire et de l'Environnement (2000). - « Ville, densités urbaines, et développement durable » Actes du Colloque, octobre 1999.

\section{RÉSUMÉS}

Un étalement urbain accompagne désormais la croissance des villes, notamment les plus grandes. Principalement expliqué par un desserrement résidentiel, il est aussi la résultante des dynamiques de centralisation de l'emploi et de spécialisation des zones d'activité à l'intérieur des aires urbaines. L'exemple dijonnais met bien en lumière ce double mouvement qui affecte les communes de sa périphérie et contribue à accroître les mobilités.

Urban sprawl arises from the growth of cities, especially of the biggest cities. This urban sprawl is accounted for mainly by residential clearing. It results also from the dynamics of centralization of employment and from specialization of activity zones in the interior of urban areas. The example of Dijon brings out this double movement which affects peripheral parishes and contributes to increase mobilities.

Mit der Stadtentwicklung ist die flächenhafte Ausdehnung des urbanen Raumes verbunden. Dies betrifft insbesondere die größten Städte. Diese entsteht durch Siedlungsauflockerung. Gleichzeitig ist sie auch eine Folge der Zentralisierung der Beschäftigung und der Spezialisierung der Gewerbegebiete. Ans Licht bringt das Beispiel Dijon diese Doppelbewegung, welche die Entwicklung der Gemeinden an den Rändern prägt und zum Anstieg der Mobilität beiträgt.

\section{INDEX}

Schlüsselwörter : expansive Suburbanisierung, Mobilität, Siedlungsauflockerung, Städtischer Raum und Knoten, Verdichtung der Beschäftigung, Wanderungen

Keywords : concentration of employment, migration, mobility, residential clearing, Urban areas and nodes, urban sprawl

Mots-clés : Aire et pôle urbains, concentration de l'emploi, desserrement résidentiel, étalement urbain, migration, mobilité 
AUTEUR

ANDRÉ LARCENEUX

Laboratoire TheMA, UMR 6039, Université de Bourgogne, 2 bd Gabriel, 21000 Dijon 